http://dx.doi.org/10.32929/2446-8355.2019v29n1p50-60

\title{
DIFERENTES DENSIDADES AMOSTRAIS NA CARACTERIZAÇÃO DA VARIABILIDADE ESPACIAL DE ATRIBUTOS GRANULOMETRICOS DE UM ARGISSOLO AMARELO DISTROCOESO TÍPICO
}

\author{
Klayton Antonio do Lago Lopes ${ }^{1 *}$, Fernando Freitas Pinto Junior ${ }^{1}$, Francisco Ivo dos Santos \\ Aguiar $^{2}$, Alex Eduardo da Silva Sousa ${ }^{3}$, Ismênia Ribeiro de Oliveira ${ }^{4}$, Jussara Silva Dantas ${ }^{5}$ \\ ${ }^{1}$ Graduando em Agronomia, Universidade Federal do Maranhão (UFMA), Centro de Ciências Agrárias e \\ Ambientais (CCAA), Chapadinha, MA. *E-mail do autor correspondente: klaytonlopes2011@ gmail.com \\ ${ }^{2}$ Mestrando em Agronomia (Ciência do Solo), Universidade Federal Rural do Rio de Janeiro (UFRRJ), \\ Seropédica, RJ. \\ ${ }^{3}$ Graduando, Engenharia Agrícola, Universidade Federal do Maranhão (UFMA), Centro de Ciências Agrárias e \\ Ambientais (CCAA), Chapadinha, MA. \\ ${ }^{4}$ Docente da Universidade Federal do Maranhão (UFMA), Centro de Ciências Agrárias e Ambientais (CCAA), \\ Dr. ${ }^{a}$ em Agronomia (Ciência do Solo), Chapadinha, MA. \\ 5 Docente da Universidade Federal de Campina Grande (UFCG), Centro de Ciências e Tecnologia \\ Agroalimentar (CCTA), Dr. ${ }^{a}$ em Agronomia (Ciência do Solo), Pombal, PB.
}

Recebido: 29/10/2018; Aceito: 17/03/2020

RESUMO: A amostragem adequada deve ser a menor possível, porém, com uma acurácia representativa, a fim de produzir resultados confiáveis. Nesse contexto, objetivou-se caracterizar a distribuição espacial dos atributos granulométricos de um Argissolo Amarelo distrocoeso típico, em função de diferentes densidades de amostragem. Foram estabelecidas três grades amostrais quadradas com 80, 100 e 121 pontos em uma área de produção de soja de 6,2 ha, na profundidade de 0,0-0,2 m. Foram analisados os atributos argila, silte e areia total. O coeficiente de variação indicou média variabilidade para argila e silte, e baixa variabilidade para areia total, nas três amostragens estudadas. O silte apresentou grau de dependência espacial forte nas três grades amostrais, enquanto a argila e areia total apresentaram grau de dependência espacial moderado nas três densidades de amostragem. $\mathrm{O}$ aumento do intervalo de amostragem e a redução do número de amostras não promoveram o aumento da raiz quadrada do erro médio e do quadrado médio do erro nas estimativas por krigagem nas diferentes intensidades amostrais estudadas. Não havendo diferença significativa entre estes parâmetros para as intensidades amostrais analisadas. Os mapas de krigagem demonstraram semelhança entre a distribuição espacial dos atributos nas três intensidades amostrais utilizadas. Em uma área de 6,2 ha, a quantidade de pontos amostrais (80, 100 e 121 pontos) não interferiu significativamente na análise da variabilidade espacial dos atributos granulométricos do solo e no detalhamento das informações dos mapas interpolados por krigagem.

Palavras-chave: Interpolação. Textura do solo. Amostragem georreferenciada. 


\title{
DIFFERENT SAMPLE DENSITIES IN THE CHARACTERIZATION OF THE SPATIAL VARIABILITY OF GRANULOMETRIC ATTRIBUTES OF A TYPICAL DYSTROCOHESIVE YELLOW ARGISSOL
}

\begin{abstract}
Adequate sampling should be as small as possible, but with a representative accuracy, in order to produce reliable results. In this context, the aim was to characterize the spatial distribution of the granulometric attributes of an Ultisol, as a function of different sampling densities. Three square sample grids with 80,100 and 121 points were established in a soybean production area of $6.2 \mathrm{ha}$, at a depth of 0.0-0.2 $\mathrm{m}$. The clay, silt and total sand attributes were analyzed. The coefficient of variation indicated average variability for clay and silt, and low variability for total sand, in the three samplings studied. The silt showed a strong degree of spatial dependence in the three sampled grids, while clay and total sand had a moderate degree of spatial dependence in the three sampling densities. The increase of the sampling interval and the reduction of the number of samples did not promote the increase of the square root of the mean error and the mean square of the error in the estimates by kriging in the different sample intensities studied. There was no significant difference between these parameters for the analyzed intensities. The kriging maps showed similarity between the spatial distribution of the attributes in the three sample intensities used. In an area of $6.2 \mathrm{ha}$, the number of sampling points $(80,100$ and 121 points) did not significantly interfere in the analysis of the spatial variability of the soil granulometric attributes and in the detailing of the information of the maps interpolated by kriging.
\end{abstract}

Key words: Interpolation. Soil texture. Georeferenced sampling.

\section{INTRODUÇÃO}

A Agricultura de Precisão (AP) está normalmente associada à utilização de equipamentos de alta tecnologia para avaliar ou monitorar uma determinada área, objetivando-se o aumento da produtividade, redução dos custos e consequentemente redução dos impactos ambientais (COELHO; SILVA, 2009). A AP se origina no mapeamento e na manipulação da variabilidade espacial das lavouras. No entanto, entende-se que ela tem várias formas de abordagem e pode ser praticada em diferentes níveis de complexidade (MOLIN; AMARAL; COLAÇO, 2015). Desta forma, o geoprocessamento tem sido uma ferramenta essencial no desenvolvimento da AP, viabilizando a coleta de solos por meio de malhas amostrais, através de amostragem georreferenciada, permitindo determinar a variabilidade espacial dos atributos do solo (CHERUBIN et al., 2015).

As técnicas geoestatísticas vêm sendo utilizadas para avaliar, de forma mais precisa, os fatores que influenciam a variabilidade dos atributos granulométricos do solo e da distribuição dos sedimentos (BOTTEGA et al., 2013; CASTIONE et al., 2015). A descrição minuciosa dessa variabilidade do solo pode apontar a ocorrência de processos capazes de influenciar na produtividade das culturas, proporcionando a identificação de locais específicos de manejo (SANCHEZ et al., 2012). 
Os métodos geoestatísticos são muito utilizados nos estudos da dependência espacial dos atributos físico-químicos do solo e da produtividade das culturas (KESTRING et al., 2015). A partir da aplicação destes métodos é possível descrever a distribuição espacial dos atributos no solo, favorecendo a construção de mapas de isolinhas com o nível de detalhe desejável para maior compreensão das relações solo-plantas (ROSA FILHO et al., 2011). Por meio do uso da geoestatística é possível modelar e visualizar espacialmente a estrutura de dependência espacial, e assim indicar os manejos adequados de forma localizada, considerando a variabilidade espacial das características do solo (MENDES; FONTES; OLIVEIRA, 2008).

Contudo, a forma e o tamanho da configuração amostral utilizada podem afetar a descrição da estrutura de dependência espacial e as estimativas espaciais de valores não amostrados e, posteriormente, a credibilidade dos resultados apresentados pelos mapas temáticos construídos (KESTRING et al., 2015). Com isso, o principal fator limitante ao uso da AP, é justamente o número de amostras necessária para formar a malha amostral ideal para representar espacialmente a distribuição dos atributos no solo (SOUZA et al., 2014). Pois, ao mesmo tempo em que a intensidade amostral deve ser suficiente para definir adequadamente a variabilidade espacial dos atributos (DEBIASI et al., 2011), a amostragem de solos muito intensa pode inviabilizar economicamente a análise da variabilidade espacial (RAGAGNIN; JUNIOR; SILVEIRA NETO, 2010).

Para as condições brasileiras, são poucas as pesquisas que buscam determinar uma dimensão ideal de grade amostral para a coleta georreferenciada de solo em áreas de AP (CHERUBIM et al., 2015). Atualmente no Brasil as áreas comerciais sob manejo da AP têm feito uso de malhas amostrais de 100x100 ou 175x175 m, que por razões econômicas geram uma amostra a cada um ou três hectare, respectivamente (CHERUBIN et al., 2014). No entanto, a intensidade amostral deve ser a menor possível e suficiente para garantir a dependência espacial e uma acurácia representativa, a fim de produzir resultados confiáveis.

Considerando que ainda não há pesquisas conclusivas sobre a quantidade de amostras necessárias para representar a distribuição espacial dos atributos dos solos no Brasil, é necessário o estudo de estratégias de amostragem para torná-las mais confiáveis e assim viabilizar o uso da agricultura de precisão. Nesse contexto, objetivou-se caracterizar a distribuição espacial dos atributos granulométricos de um Argissolo Amarelo distrocoeso típico, em função de diferentes densidades de amostragem.

\section{MATERIAL E MÉTODOS}

O estudo foi desenvolvido no município de Brejo, MA, microrregião de Chapadinha, MA, nas coordenadas geográficas de $03^{\circ} 36^{\prime} \mathrm{S}$ e $42^{\circ} 52^{\prime} \mathrm{W}$. O clima, segundo a classificação de Köppen, é do tipo tropical quente e úmido ( $\mathrm{Aw}$ ), com temperatura média anual superior a $27^{\circ} \mathrm{C}$ e precipitação pluvial média anual de $1.835 \mathrm{~mm}$. O solo foi classificado como Argissolo Amarelo distrocoeso típico, formado por sedimentos areno argilosos do Grupo Barreiras, característico da unidade geomorfológica Tabuleiros Costeiros (ATLAS DO MARANHÃO, 2002). Foram estabelecidas três grades amostrais quadradas com 80, 100 e 121 pontos em uma área de produção de soja de 6,2 ha, na profundidade de 0,0-0,2 m. 
(a)
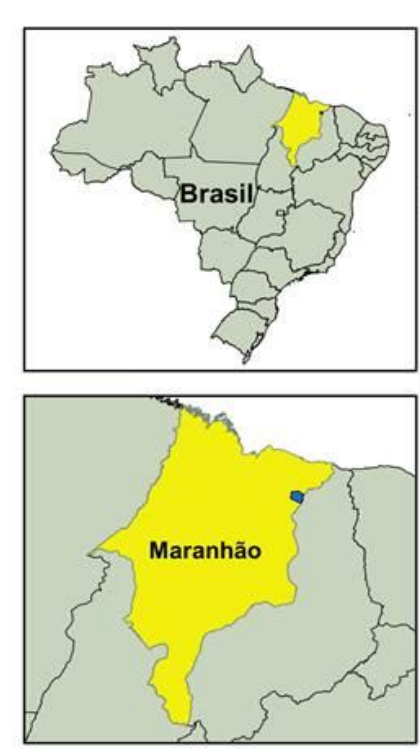

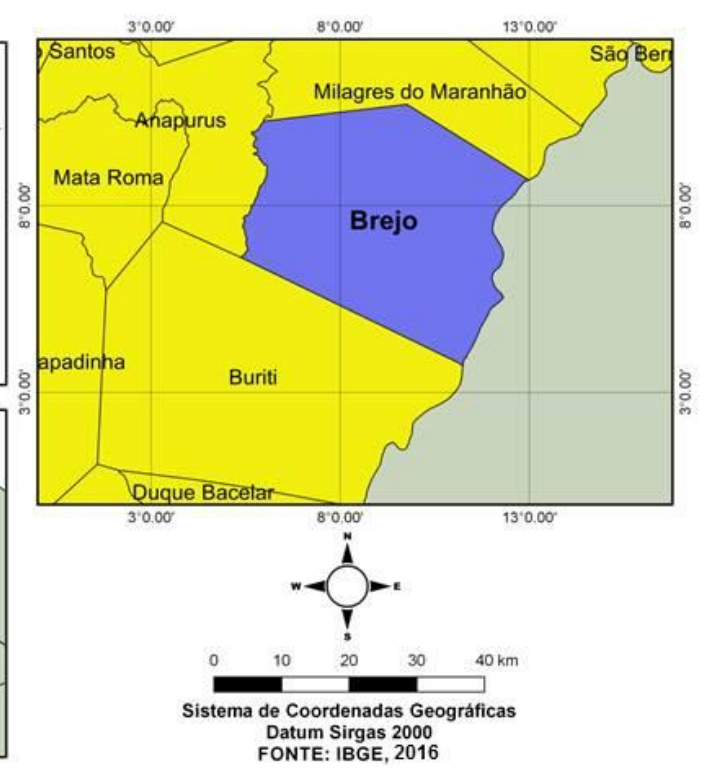

(b)
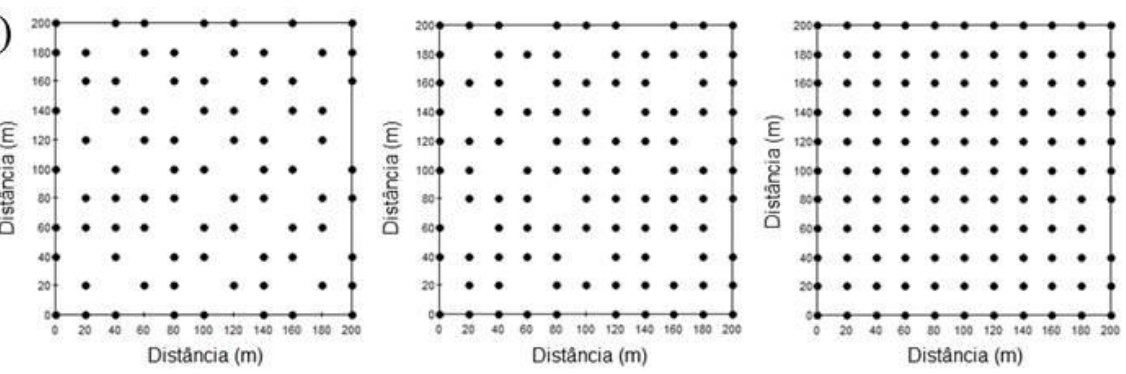

Figura 1. Localização da área experimental (a); e grades amostrais de 80, 100 e 121 pontos, respectivamente. Location of the experimental area (a); and sample grids of 80, 100 and 121 points, respectively.

Fonte: Autoria própria. Own authorship.

Foram analisados os atributos argila, silte e areia total (AT) por meio do método da Embrapa (1997). A estatística descritiva (média, desvio padrão, coeficiente de variação, mínimo, máximo, assimetria e curtose) dos valores observados dos atributos, foi realizada com o objetivo de obter informações para identificar tendência, dispersão e forma de distribuição dos dados. Para verificar a hipótese de normalidade dos dados foi utilizado o teste de Kolmogorov-Smirnov, a nível de 5\% de probabilidade.

A classificação do grau de dependência espacial (GDE) foi feita com base na razão entre o efeito pepita e o patamar $\left(\mathrm{C}_{0} / \mathrm{C}_{0}+\mathrm{C}_{1}\right)$, sendo considerada forte, quando $\leq 25 \%$; moderado, quando $25 \%<\mathrm{GDE} \leq 75 \%$; e fraco quando GDE $>75 \%$ (CAMBARDELLA et al., 1994).

A variabilidade espacial dos atributos argila, silte e AT foram caracterizadas por meio do semivariograma, determinado por meio do cálculo da variância em razão da distância de separação entre amostras por meio da Equação 1.

$$
\hat{\gamma}(h)=\frac{1}{2 N(h)} \sum_{i=1}^{N(h)}\left[z\left(x_{i}\right)-z\left(x_{i}+h\right)\right]^{2}
$$


em que, $\hat{\gamma}(h)$ é a semivariância experimental para uma distância de separação $h, z(x i)$ é o valor da propriedade no ponto $i$, e $N(h)$ é o número de pares de pontos separados pela distância $h$. Conforme o ajuste do modelo matemático, foram definidos os parâmetros do modelo teórico para o semivariograma (o efeito pepita, $\mathrm{C}_{0}$; variância estrutural, $\mathrm{C}_{1}$; patamar, $\mathrm{C}_{0}+\mathrm{C}_{1}$ e o alcance, a). $\mathrm{O}$ efeito pepita é o valor da semivariância para distância zero e representa o componente da variação ao acaso; o patamar é o valor da semivariância em que a curva estabiliza sobre um valor constante; o alcance é a distância da origem até onde o patamar atinge valores estáveis, expressando a distância além da qual as amostras não são correlacionadas (SOARES, 2006). A escolha dos modelos teóricos dos semivariogramas e o ajuste dos seus parâmetros foi feita observando o melhor coeficiente de correlação obtidos pela técnica de validação cruzada e o maior coeficiente de determinação $\left(R^{2}\right)$, sendo os valores de $\mathrm{R}^{2}$ mais próximos de 1 aqueles que caracterizam o modelo mais eficiente para expressar o fenômeno estudado.

Após a modelagem dos semivariogramas, foi utilizada a técnica krigagem ordinária (KO) para a interpolação de valores em locais não mostrados (SOARES, 2006). Essa técnica é baseada em uma média móvel ponderada das amostras vizinhas, obtida pela Equação 2.

$$
\hat{z}\left(x_{0}\right)=\sum_{i=1}^{N} \lambda_{i} z\left(x_{i}\right), \operatorname{com}, \sum_{i=1}^{N} \lambda_{i}=1
$$

na qual $\hat{z}\left(x_{0}\right)$, é o valor estimado no ponto $0 ; N$ é o número de valores utilizados na estimação; $\lambda$ é o peso associado a cada valor observado, e $z(x i)$ é o valor observado no ponto $i$. Os pesos $(\lambda i)$ de cada vizinho são determinados utilizando o modelo do semivariograma ajustado, resultando em uma estimativa de variância mínima (SOARES, 2006).

Para analisar a precisão da interpolação foram utilizados a raiz quadrada do erro médio (RQEM) e o quadrado médio do erro (QME) (KRAVCHENKO, 2003).

Toda modelagem geoestatística foi realizada utilizando o programa GS+ (ROBERTSON, 1998). O programa Surfer (SURFER, 1999) foi utilizado para a elaboração dos mapas de distribuição espacial dos atributos.

\section{RESULTADOS E DISCUSSÃO}

Os resultados referentes à estatística descritiva (Tabela 1) indicaram normalidade dos dados para os atributos argila, em ambas as intensidades de amostragem, e para areia total na amostragem de 121 pontos, conforme o teste de Kolmogorov-Smirnov, a nível de 5\% de probabilidade. Que pode ser observada através dos valores do coeficiente de assimetria (Ass.) e do coeficiente de curtose (Curt.) próximos à zero.

Em relação aos atributos silte e areia total, todas as intensidades de amostragem apresentaram Ass. de moderada $(0,15<\mid$ Ass. $\mid<1)$ a Forte $(1<\mid$ Ass. $)$ (CRESPO, 2002). Embora esses valores indiquem distribuições assimétricas para as três malhas amostrais dos atributos silte e areia total, os valores da média e mediana de todos os atributos analisados são próximos, o que indica que os dados não apresentam assimetria acentuada. 
Tabela 1. Estatística descritiva dos atributos granulométricos do solo nas diferentes intensidades de amostragem. Descriptive statistics of soil granulometric attributes at different sampling intensities.

\begin{tabular}{cccccccccc}
\hline Pontos & Média & Mediana & DP & CV & Mín. & Máx. & Ass. & Curt. & P-valor \\
\hline \multicolumn{8}{c}{ Argila, $\mathrm{g} \mathrm{kg}^{-1}$} \\
\hline 80 & 144,10 & 145,75 & 23,20 & 16,10 & 92,00 & 216,50 & 0,09 & 0,64 & $>0,150^{*}$ \\
100 & 145,07 & 146,25 & 22,23 & 15,32 & 86,00 & 216,50 & $-0,02$ & 0,75 & $>0,150^{*}$ \\
121 & 145,41 & 146,25 & 22,82 & 15,69 & 86,00 & 216,50 & 0,00 & 0,58 & $>0,150^{*}$ \\
\hline \multicolumn{8}{c}{ Silte, $\mathrm{g} \mathrm{kg}^{-1}$} \\
\hline 80 & 80,63 & 73,50 & 19,40 & 24,06 & 48,50 & 129,50 & 0,90 & $-0,24$ & $<0,010$ \\
100 & 80,44 & 72,75 & 20,22 & 25,14 & 48,50 & 137,50 & 0,99 & $-0,01$ & $<0,010$ \\
121 & 80,85 & 73,50 & 20,15 & 24,92 & 48,50 & 137,50 & 1,02 & 0,09 & $<0,010$ \\
\hline \multicolumn{8}{c}{ Areia Total, $\mathrm{g} \mathrm{kg}^{-1}$} \\
\hline 80 & 775,28 & 777,00 & 25,77 & 3,32 & 683,00 & 824,00 & $-0,90$ & 2,04 & 0,045 \\
100 & 774,48 & 776,50 & 25,60 & 3,31 & 683,00 & 824,00 & $-1,06$ & 2,36 & 0,040 \\
121 & 773,74 & 775,50 & 25,95 & 3,35 & 683,00 & 824,00 & $-0,90$ & 1,73 & $0,083^{*}$ \\
\hline
\end{tabular}

DP = Desvio-padrão; CV= Coeficiente de variação (\%); Mín= Mínimo; Máx= Máximo; Ass.= Coeficiente de assimetria; Curt. $=$ Coeficiente de curtose; P-valor para o teste de normalidade Kolmogorov-Smirnov a nível de 5\% de significância; $(*)$ Variável com distribuição normal. $S D=$ standard deviation; $C V=$ Coefficient of variation (\%); Min = Minimum; Max = Maximum; Ass. = Asymmetry coefficient $;$ Kurt = Coefficient of kurtosis; $P$-value for the Kolmogorov-Smirnov normality test at 5\% level of significance; (*) Variable with normal distribution.

Fonte: Autoria própria. Own authorship.

Conforme Cressie (1991) a condição de normalidade na distribuição dos dados não é uma exigência para a análise geoestatística. Porém as distribuições dos atributos analisados não podem apresentar uma assimetria muito acentuada. Uma vez que a distribuição de dados com calda muito alongada poderia comprometer a análise, principalmente em estimativas que são baseadas na média como a krigagem (ISAAKS; SRIVASTAVA, 1989).

As três densidades de amostragem promoveram similaridade das estatísticas média e mediana entre as diferentes intensidades amostrais. Conforme observado por Souza et al. (2014), os quais relatam que a semelhança das estatísticas descritivas entre diferentes intensidades de amostragem representam uma mesma população (SOUZA et al., 2014).

O coeficiente de variação (CV) indicou média variabilidade (CV de $12 \%$ a $60 \%$ ) para argila e silte, e baixa variabilidade $(\mathrm{CV}<12 \%)$ para areia total, nas três amostragens estudadas (WARRICK; NIELSEN, 1980). Assemelhando-se aos resultados obtidos por Lima et al. (2015) e Tavares et al. (2012), os quais observaram média variabilidade para argila e silte, e baixa variabilidade para areia. No entanto, Souza et al. (2014) verificaram baixa variabilidade para argila e areia nas diferentes densidades amostrais.

O alcance representa a distância em que os pontos amostrais estão correlacionados espacialmente entre si, indicando que pontos localizados numa área de raio igual ao alcance são mais homogêneos entre si. Dessa forma, o silte apresentou menores valores de alcance nas 
três malhas amostrais avaliadas, indicando que estes atributos apresentam uma maior variabilidade no solo estudado.

O silte apresentou GDE forte nas três grades amostrais, ao passo que argila e areia total apresentaram GDE moderado em ambas às densidades de amostragem (Tabela 2). Para Souza et al. (2014) as diferentes densidades amostrais não influenciaram de forma significativa na dependência espacial dos atributos granulométricos estudados, uma vez que a argila e areia apresentaram grau de dependência espacial moderado.

Os semivariogramas dos atributos estudados, nas diferentes intensidades de amostragem, foram ajustados ao modelo esférico, sendo este modelo o mais utilizado para descrever o comportamento de atributos do solo (CAMBARDELLA et al., 1994).

Tabela 2. Modelos e parâmetros estimados dos semivariogramas experimentais para os atributos granulométricos em diferentes intensidades de amostragem. Models and estimated parameters of the experimental semivariograms for the granulometric attributes at different sampling intensities.

\begin{tabular}{cccccccc}
\hline Pontos & Modelo & $\mathrm{C}_{0}$ & $\mathrm{C}_{0}+\mathrm{C}_{1}$ & Alcance & $\mathrm{GDE}(\%)$ & $\mathrm{R}^{2}$ & $\mathrm{SQR}$ \\
\hline \multicolumn{7}{c}{ Argila, $\mathrm{g} \mathrm{kg}^{-1}$} \\
\hline 80 & Esférico & 319,85 & 507,02 & 62,15 & 63,08 & 0,835 & $1,5 \mathrm{E}+03$ \\
100 & Esférico & 192,14 & 467,11 & 52,36 & 41,13 & 0,789 & $3,5 \mathrm{E}+03$ \\
121 & Esférico & 274,47 & 489,83 & 62,42 & 56,03 & 0,961 & $5,9 \mathrm{E}+02$ \\
\hline \multicolumn{7}{c}{ Silte, $\mathrm{g} \mathrm{kg}^{-1}$} \\
\hline 80 & Esférico & 1,00 & 348,54 & 38,13 & 0,29 & 0,637 & $5,6 \mathrm{E}+03$ \\
100 & Esférico & 83,04 & 396,92 & 48,14 & 20,92 & 0,755 & $3,7 \mathrm{E}+03$ \\
121 & Esférico & 0,01 & 0,05 & 49,26 & 15,86 & 0,848 & $5,0 \mathrm{E}-05$ \\
\hline \multicolumn{7}{c}{ Areia Total, $\mathrm{g} \mathrm{kg}^{-1}$} \\
\hline 80 & Esférico & 396,31 & 573,57 & 55,72 & 69,10 & 0,955 & $3,87 \mathrm{E}+02$ \\
100 & Esférico & 450,04 & 690,15 & 56,73 & 65,21 & 0,610 & $5,98 \mathrm{E}+03$ \\
121 & Esférico & 457,90 & 692,67 & 48,79 & 66,11 & 0,761 & $2,66 \mathrm{E}+03$ \\
\hline
\end{tabular}

$\mathrm{C}_{0}=$ Efeito pepita; $\mathrm{C}_{0}+\mathrm{C} 1=$ Patamar; GDE $=$ Grau de Dependência Espacial $\left(\mathrm{C}_{0} /\left(\mathrm{C}_{0}+\mathrm{C} 1\right) * 100 ; \mathrm{R}^{2}=\right.$ Coeficiente de Determinação; $\mathrm{SQR}=$ Soma dos Quadrados dos Resíduos. $C_{o}=$ Nugget effect; $C_{o}+C 1=$ Porch; $G D E=$ Degree of Spatial Dependence $\left(C_{o} /\left(C_{o}+C 1\right) * 100, R^{2}=\right.$ Determination Coefficient, SQR $=$ Sum of Squares of Residues.

Fonte: Autoria própria. Own authorship.

O maior coeficiente de determinação $\left(\mathrm{R}^{2}\right)$ e a menor soma dos quadrados dos resíduos (SQR) foram encontrados na grade amostral de 121 pontos para os atributos argila e silte, e na amostragem de 80 pontos para areia total. Desta forma, a redução do número de amostras não promoveu um decréscimo no $\mathrm{R}^{2}$ e nem um aumento no $\mathrm{SQR}$ para os atributos analisados. Não havendo diferença significativa entre estes parâmetros para as intensidades amostrais analisadas.

Com o aumento do número de amostras, a raiz quadrada do erro médio (RQEM) e o quadrado médio do erro (QME), não apresentaram diferenças significativas entre as diferentes 
intensidades amostrais estudadas, uma vez que os valores encontram-se próximos entre si (Tabela 3). Diferentemente do observado por Souza et al. (2014), em que o aumento do intervalo de amostragem e a redução do número de amostras promoveram acréscimos significativos nos valores da raiz quadrada do erro médio e do quadrado médio do erro nas estimativas por krigagem nas duas áreas estudadas.

Tabela 3. Raiz quadrada do erro médio (RQEM) e quadrado médio do erro (QME) da validação cruzada da análise geoestatística e interpolação por krigagem dos atributos granulométricos do solo nas diferentes intensidades de amostragem. Square root mean error (SRME) and mean square error (MSE) of the cross validation of the geostatistical analysis and interpolation by kriging the soil granulometric attributes at the different sampling intensities.

\begin{tabular}{ccccc}
\hline & Pontos & 80 & 100 & 121 \\
\cline { 2 - 4 } & \multicolumn{4}{c}{ Argila, $\mathrm{g} \mathrm{kg}^{-1}$} \\
\cline { 2 - 4 } RQEM & 21,5 & 20,64 & 20,68 \\
QME & 462,26 & 426,03 & 427,75 \\
\hline \multicolumn{4}{c}{ Silte, $\mathrm{g} \mathrm{kg}^{-1}$} \\
\cline { 2 - 4 } & RQEM & 17,12 & 17,89 & 16,82 \\
QME & 293,04 & 319,94 & 282,93 \\
\hline \multicolumn{4}{c}{ Areia Total, $\mathrm{g} \mathrm{kg}^{-1}$} \\
\hline Fonte: Autoria própria. Own authorship. & 23,42 & 25,26 & 25,73 \\
& RQEM & 23,407 & 638,20 & 661,95 \\
\hline
\end{tabular}

Os menores valores da RQEM e do QME dos atributos argila, silte e areia total foram encontrados nas grades amostrais de 100, 121 e 80 pontos, respectivamente. Não havendo diferença significativa entre as três intensidades amostrais analisadas, em função destes parâmetros estudados. Souza et al. (2014) observou que os variogramas construídos com 105 pontos não diferiram dos variogramas com 208 pontos na área estudada, refletindo na recomendação mínima de 100 pontos para viabilizar o uso da geoestatística e da krigagem. Dias et al. (2017) ao avaliar duas grades amostrais, observou que a grade de 49 pontos demonstrou grande potencial em detectar diferentes escalas de variabilidade espacial dos atributos físicos, em relação a grade de 16 pontos.

Cherubim et al. (2015) relatam que os mapas de variabilidade do solo tornam-se mais similares à medida que aumenta o numero de pontos da grade amostral. No entanto, os mapas de krigagem (Figura 2) demonstraram semelhança entre a distribuição espacial dos atributos nas três intensidades amostrais avaliadas. Desta forma, as intensidades amostrais estudadas não promoveram a dissimilaridades entres a distribuição espacial dos atributos estudados.

Os atributos argila e silte apresentaram a menor e maior variabilidade entre as diferentes grades amostrais estudadas, respectivamente. 
(a)

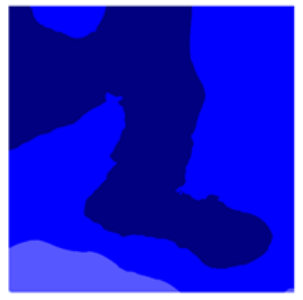

(a)

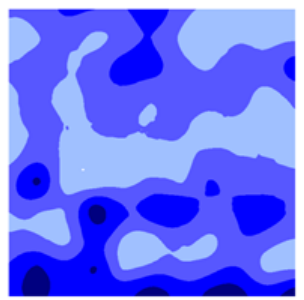

(a)

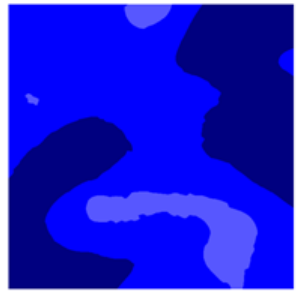

(b)

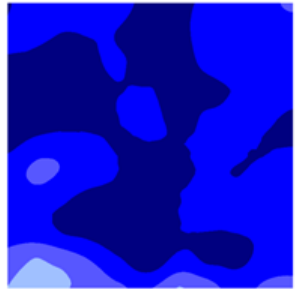

Argila, $\mathrm{g} \mathrm{kg}^{-1}$ (c)

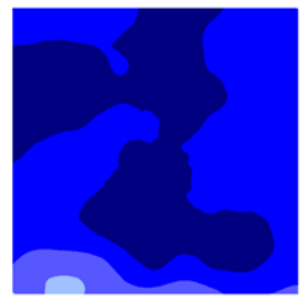

(c)

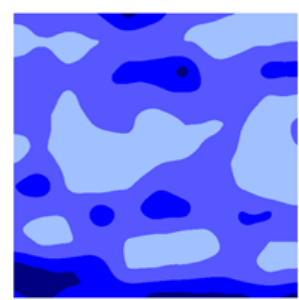

(b)

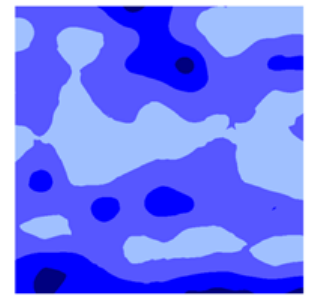

Silte, $\mathrm{g} \mathrm{kg}^{-1}$ (b)

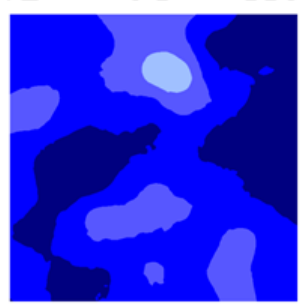

Areia Total, $\mathrm{g} \mathrm{kg}^{-1}$

$\begin{array}{llll}737 & 751 & 765 & 779\end{array}$

(c)

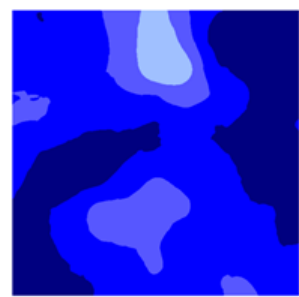

Figura 2. Mapas de krigagem dos atributos granulométricos do solo nas diferentes intensidades de amostragem: 80 pontos (a); 100 pontos (b); 121 pontos (c). Kriging maps of soil granulometric attributes at different sampling intensities: 80 points (a); 100 points (b); 121 points $(c)$.

Fonte: Autoria própria. Own authorship.

\section{CONCLUSÃO}

Em uma área de 6,2 ha, a quantidade de pontos amostrais (80, 100 e 121 pontos) não interferiu significativamente na análise da variabilidade espacial dos atributos granulométricos do solo e no detalhamento das informações dos mapas interpolados por krigagem.

\section{REFERÊNCIAS BIBLIOGRÁFICAS}

ATLAS DO MARANHÃO. Gerência de Planejamento e Desenvolvimento Econômico/Laboratório de Geoprocessamento - UEMA. 2. ed. São Luís: GEPLAN, 2002. $44 \mathrm{p}$. 
BOTTEGA, E. L.; QUEIROZ, D. M.; PINTO, F. A. C.; SOUZA, C. M. A. Variabilidade espacial de atributos do solo em sistema de semeadura direta com rotação de culturas no cerrado brasileiro. Revista Ciência Agronômica, Fortaleza, v. 44, n. 1, p.1-9, 2013.

CAMBARDELLA, C. A.; MOORMAN, T. B.; NOVAK, J. M.; PARKIN, T. B.; KARLEN, D. L.; TURCO, R. F.; KONOPKA, A. E. Field-scale variability of soil properties in central Iowa soils. Soil Science Society of American Jounal, Madison, v. 58, n. 5, p.1501-1511, 1994.

CASTIONE, G. A. F.; SOUZA, Z. M.; SIlvA, R. B.; CAMPOS, M. C. C.; CUNHA, J. M. Variabilidade espacial da textura do solo em área irrigada por pivô central em diferentes posições na paisagem. Revista Agroambiente, Boa Vista, v. 9, n. 3, p.219-226, 2015.

CHERUBIN, M. R.; SANTI, A. L.; EITELWEIN, M. T.; AMADO, T. J. C.; SIMON, D. H.; DAMIAN, J. M. Dimensão da malha amostral para caracterização da variabilidade espacial de fósforo e potássio em Latossolo Vermelho. Pesquisa Agropecuária Brasileira, Brasília, v. 50, n. 2, p.168-177, 2015.

CHERUBIN, M. R.; SANTI, A. L.; EITELWEIN, M. T.; MENEGOL, D. R.; ROS, C. O.; CASTRO PIAS, O. H.; BERGHETTI, J. Eficiência de malhas amostrais utilizadas na caracterização da variabilidade espacial de fósforo e potássio. Ciência Rural, Santa Maria, v. 44, n. 3, p.425-432, 2014.

COELHO, J. P. C.; SILVA, J. R. M. Agricultura de precisão. Lisboa: AJAP, 2009. 141 p.

CRESPO, A. A. Estatística fácil. 17. ed. São Paulo: Saraiva, 2002. 224 p.

CRESSIE, N. Statistics for spatial data. New York: John Wiley, 1991. 900 p.

DEBIASI, H.; FRANCHINI, J. C.; OLIVEIRA, F. A.; MACHADO, T. M. Ajuste de grades amostrais para o mapeamento da resistência à penetração de um Latossolo Bruno. In: INAMASU, R. Y.; NAIME, J. M.; RESENDE, A. V.; BASSOI, L. H.; BERNARDI, A. C. C. (ed.). Agricultura de precisão: um novo olhar. São Carlos: Embrapa Instrumentação Agropecuária, 2011. cap. 2, p.138-142.

DIAS, F. P. M.; CASTRO, J. R.; NUNES, F. J.; NONATO, A. C. R.; BOTELHO JUNIOT, F.; FÉ, J. A. R.; SANTOS, D. N.; NÓBREGA, J. C. A. Eficiência de malhas amostrais na caracterização da variabilidade espacial de atributos físicos do solo. Anuário do Instituto de Geociências, Rio de Janeiro, v. 40, n. 2, p.31-36, 2017.

EMPRESA BRASILEIRA DE PESQUISA AGROPECUÁRIA - EMBRAPA. Manual de métodos de análises do solo. 2. ed. Rio de Janeiro, 1997. 212 p.

ISAAKS, E. H.; SRIVASTAVA, R. M. A Introduction to applied geostatistics. Oxford University Press, 1989. 592 p.

KESTRING, F. B. F.; GUEDES, L. P. C.; BASTIANI, F.; URIBE-OPAZO, M. A. Comparação de mapas temáticos de diferentes grades amostrais para a produtividade da soja. Engenharia Agrícola, Jaboticabal, v. 35, n. 4, p.733-743, 2015.

KRAVCHENKO, A. N. Influence of spatial structure on accuracy of interpolation methods. Soil Science Society of American Journal, Madison, v. 67, n. 5, p.1564-1571, 2003. 
LIMA, F. V.; SILVINO, G. S.; MELO, R. S.; LIRA, E. C.; RIBEIRO, T. S. Variabilidade espacial de atributos físicos do solo em área de encosta sob processo de degradação. Revista Caatinga, Mossoró, v. 28, n. 4, p.53-63, 2015.

MENDES, A. M. S.; FONTES, R. L. F.; OLIVEIRA, M. Variabilidade espacial da textura de dois solos do Deserto Salino, no Estado do Rio Grande do Norte. Revista Ciência Agronômica, Fortaleza, v. 39, n. 1, p.19-27, 2008.

MOLIN, J. P.; AMARAL, L. R.; COLAÇO, A. F. Agricultura de precisão. São Paulo: Oficina de Textos, 2015. 223 p.

OLIVEIRA, I. A.; CAMPOS, M. C. C.; SOARES, M. D. R.; AQUINO, R. E.; MARQUES JÚNIOR, J. \& NASCIMENTO, E. P. Variabilidade espacial de atributos físicos em um Cambissolo Háplico, sob diferentes usos na região sul do Amazonas. Revista Brasileira de Ciência do Solo. Viçosa, v. 37, n. 4, p.1103-1112, 2013.

RAGAGNINI, V. A.; JUNIOR, D. G. S.; SILVEIRA NETO, A. N. Recomendação de calagem a taxa variada sob diferentes intensidades de amostragem. Revista Brasileira de Engenharia Agrícola e Ambiental, Campina Grande, v. 14, n. 6, p.600-607, 2010.

ROBERTSON, G. P. GS+: Geostatistics for the environmental Sciences-GS+ User's guide. Plainwell, Gamma Design Software, 1998. 152 p.

ROSA FILHO, G.; CARVALHO, M. P.; MONTANARI, R. M.; SILVA, J. M.; SIQUEIRA, G. M.; ZAMBIANCO, E. C. Variabilidade espacial de propriedades dendrométricas do eucalipto e de atributos físicos de um Latossolo Vermelho. Bragantia, Campinas, v. 70, n. 2, p.439-446, 2011.

SANCHEZ, R. B.; MARQUES JUNIOR, J.; PEREIRA, G. T.; BARACAT NETO, J.; SIQUEIRA, D. S.; SOUZA, Z. M. Mapeamento das formas do relevo para estimativa de custos de fertilização em cana-de-açúcar. Engenharia Agrícola, Jaboticabal, v. 32, n. 2, p.280-292, 2012.

SOARES, A. Geoestatística para ciências da terra e do ambiente. 2. ed. Lisboa: IST Press, 2006. 214 p.

SOUZA, Z. M.; SOUZA, G. S.; MARQUES JÚNIOR, J.; PEREIRA, G. T. Número de amostras na análise geoestatística e na krigagem de mapas de atributos do solo. Ciência Rural, Santa Maria, v. 44, n. 2, p.261-268, 2014.

SURFER for windows. Realese 7.0. Contouring and 3D surface mapping for scientist's engineers. User's guide. New York: Golden Software, 1999. 619 p.

TAVARES, U. E. ROLIM, M. M.; PEDROSA, E. M. R.; MONTENEGRO, A. A. A.; MAGALHÃES, A. G.; BARRETO, M. T. L. Variabilidade espacial de atributos físicos e mecânicos de um Argissolo sob cultivo de cana-de-açúcar. Revista Brasileira de Engenharia Agrícola e Ambiental, Campina Grande, v. 16, n. 11, p.1206-1214, 2012.

WARRICK, A. W.; NIELSEN, D. R. Spatial variability of soil physical properties in the field. In: HILLEL, D. (Ed.). Applications of soil physics. New York: Academic, 1980. p. 319-344. 